\title{
Composition Operators on Cesàro Function Spaces
}

\author{
Kuldip Raj, Suruchi Pandoh, and Seema Jamwal \\ School of Mathematics, Shri Mata Vaishno Devi University, Katra 182320, India \\ Correspondence should be addressed to Kuldip Raj; kuldeepraj68@rediffmail.com
}

Received 17 May 2013; Accepted 20 November 2013; Published 30 January 2014

Academic Editor: Satit Saejung

Copyright (C) 2014 Kuldip Raj et al. This is an open access article distributed under the Creative Commons Attribution License, which permits unrestricted use, distribution, and reproduction in any medium, provided the original work is properly cited.

The compact, invertible, Fredholm, and closed range composition operators are characterized. We also make an effort to compute the essential norm of composition operators on the Cesàro function spaces.

\section{Introduction and Preliminaries}

Let $(X, s, \mu)$ be a $\sigma$-finite measure space and let $L^{0}=L^{0}(X)$ denote the set of all equivalence classes of complex valued measurable functions defined on $X$, where $X=[0,1]$ or $X=[0, \infty)$. Then, for $1 \leq p<\infty$, the Cesàro function space is denoted by $\operatorname{Ces}_{p}(X)$ and is defined as

$$
\begin{aligned}
& \operatorname{Ces}_{p}(X) \\
& \quad=\left\{f \in L^{0}(X): \int_{X}\left(\frac{1}{x} \int_{0}^{x}|f(t)| d \mu(t)\right)^{p} d \mu(x)<\infty\right\} .
\end{aligned}
$$

The Cesàro function space $\operatorname{Ces}_{p}(X)$ is a Banach space under the norm

$$
\|f\|=\left(\int_{X}\left(\frac{1}{x} \int_{0}^{x}|f(t)| d \mu(t)\right)^{p} d \mu(x)\right)^{1 / p} ;
$$

see $[1]$.

The Cesàro functions spaces $\operatorname{Ces}_{p}[0, \infty)$ for $1 \leq p \leq \infty$ were considered by Shiue [2], Hassard and Hussein [3], and Sy et al. [4]. The space $\mathrm{Ces}_{\infty}[0,1]$ appeared already in 1948 and it is known as the Korenblyum, Krein, and Levin space $K$ (see $[5,6]$ ). Recently, in [7], it is proved that, in contrast to Cesàro sequence spaces, the Cesàro function spaces $\operatorname{Ces}_{p}(X)$ on both $X=[0,1]$ and $X=[0, \infty)$ for $1<p<\infty$ are not reflexive and they do not have the fixed point property. In [8], Astashkin and Maligranda investigated Rademacher sums in $\mathrm{Ces}_{p}[0,1]$ for $1 \leq p \leq \infty$. The description is different for $1 \leq p<\infty$ and $p=\infty$.
Let $T: X \rightarrow X$ be a nonsingular measurable transformation; that is, $\mu T^{-1}(A)=\mu\left(T^{-1}(A)\right)=0$, for each $A \in s$, whenever $\mu(A)=0$. This condition means that the measure $\mu T^{-1}$ is absolutely continuous with respect to $\mu$. Let $f_{0}=d \mu T^{-1} / d \mu$ be the Radon-Nikodym derivative. In addition, we assume that $f_{0}$ is almost everywhere finite valued or equivalently that $\left(X, T^{-1}(s), \mu\right)$ is $\sigma$-finite. An atom of the measure $\mu$ is an element $A \in s$ with $\mu(A)>0$ such that, for each $F \in s$, if $F \subset A$, then either $\mu(F)=0$ or $\mu(F)=\mu(A)$. Let $A$ be an atom. Since $\mu$ is $\sigma$-finite, it follows that $\mu(A)<\infty$. Also every $s$-measurable function $f$ on $X$ is constant almost everywhere on $A$. It is a well-known fact that every sigma finite measure space $(X, s, \mu)$ can be decomposed into two disjoint sets $X_{1}$ and $X_{2}$ such that $\mu$ is atomic over $X_{1}$ and $X_{2}$ is a countable collection of disjoint atoms (see [9]).

Any nonsingular measurable transformation $T$ induces a linear operator $C_{T}$ from $\operatorname{Ces}_{p}(X)$ into the linear space of equivalence classes of $s$-measurable functions on $X$ defined by $C_{T} f=f \circ T, f \in \operatorname{Ces}_{p}(X)$. Hence, the nonsingularity of $T$ guarantees that the operator $C_{T}$ is well defined. If $C_{T}$ takes $\operatorname{Ces}_{p}(X)$ into itself, then we call that $C_{T}$ is a composition operator on $\operatorname{Ces}_{p}(X)$. By $B\left(\operatorname{Ces}_{p}(X)\right)$, we denote the set of all bounded linear operators from $\operatorname{Ces}_{p}(X)$ into itself.

So far as we know, the earliest appearance of a composition transformation was in 1871 in a paper of Schrljeder [10], where it is asked to find a function $f$ and a number $\alpha$ such that

$$
(f \circ T)(z)=\alpha f(z)
$$


for every $z$, in an appropriate domain, whenever the function $T$ is given. If $z$ varies in the open unit disk and $T$ is an analytic function, then a solution is obtained by Köenigs [11]. In 1925, these operators were employed in Littlewood's subordination theory [12]. In the early 1930s, the composition operators were used to study problems in mathematical physics and especially classical mechanics; see Koopman [13]. In those days, these operators were known as substitution operators. The systematic study of composition operators has relatively a very short history. It was started by Nordgren in 1968 in his paper [14]. After this, the study of composition operators has been extended in several directions by several mathematicians. For more details on composition operators, see [15-27] and references therein.

Associated with each $\sigma$-finite subalgebra $s_{0} \subset s$, there exists an operator $E=E^{s_{0}}$, which is called conditional expectation operator; on the set of all nonnegative measurable functions $f$ or for each $f \in L^{0}(X, s, \mu)$, the operator $E$ is uniquely determined by the following conditions:

(i) $E(f)$ is $s_{0}$-measurable;

(ii) if $A$ is any $s_{0}$-measurable set for which $\int_{A} f d \mu$ exists, we have $\int_{A} f d \mu=\int_{A} E(f) d \mu$.

The expectation operator $E$ has the following properties:

(a) $E(f \cdot g \circ T)=E(f) \cdot(g \circ T)$;

(b) if $f \geq g$ almost everywhere, then $E(f) \geq E(g)$ almost everywhere;

(c) $E(1)=1$;

(d) $E(f)$ has the form $E(f)=g \circ T$ for exactly one $\sigma$ measurable function $g$, in particular, $g=E(f) \circ T^{-1}$ is a well-defined measurable function;

(e) $|E(f g)|^{2} \leq\left(E|f|^{2}\right)\left(E|g|^{2}\right)$; this is a Cauchy-Schwartz inequality for conditional expectation;

(f) for $f>0$ almost everywhere, $E(f)>0$ almost everywhere.

For deeper study of properties of $E$, see [28].

Let $\mathscr{B}$ be a Banach space and let $\mathscr{K}$ be the set of all compact operators on $\mathscr{B}$. For $U \in \mathscr{L}(\mathscr{B})$, the Banach algebra of all bounded linear operators on $\mathscr{B}$ into itself, the essential norm of $U$ means the distance from $U$ to $\mathscr{K}$ in the operator norm; namely,

$$
\|U\|_{e}=\inf \{\|U-S\|: S \in \mathscr{K}\} .
$$

Clearly, $U$ is compact if and only if $\|U\|_{e}=0$. As seen in [29], the essential norm plays an interesting role in the compact problems of concrete operators. Many people have computed the essential norm of various concrete operators. For the study of essential norm of composition operators, see [30-33] and reference therein.

The question of actually calculating the norm and essential norm of composition operators on Cesàro function spaces is not a trivial one. In spite of the difficulties associated with computing the essential norm exactly, it is often possible to find upper and lower bound for the essential norm of
$C_{T}: \operatorname{Ces}_{p}(X) \rightarrow \operatorname{Ces}_{p}(X)$ under certain conditions on $p$ and $X$.

The main purpose of this paper is to characterize the boundedness, compactness, closed range, and Fredholmness of composition operators on Cesàro function spaces. We also make an effort to compute the essential norm of composition operators in Section 3 of this paper.

\section{Composition Operators}

In this section of the paper, we will investigate the necessary and sufficient condition for a composition operator to be bounded.

Theorem 1. Let $(X, s, \mu)$ be a $\sigma$-finite measure space and let $T: X \rightarrow X$ be nonsingular measurable transformation. Then, $T$ induces a composition operator $C_{T}$ on $\operatorname{Ces}_{p}(X)$ if and only if there exists $M>0$ such that $\mu T^{-1}(E) \leq M \mu(E)$ for every $E \in s$. Moreover,

$$
\left\|C_{T}\right\|=\sup _{0<\mu(E)<\infty}\left(\left(\frac{\mu\left(T^{-1}(E)\right)}{\mu(E)}\right)^{p}\right)^{1 / p} .
$$

Proof. Suppose that $C_{T}$ is a composition operator. If $E \in s$ such that $\mu(E)<\infty$, then $\chi_{E} \in \operatorname{Ces}_{p}(X)$ and

$$
\begin{aligned}
& \mu T^{-1}(E)=\left\|C_{T} \chi_{E}\right\|^{p} \leq\left\|C_{T}\right\|^{p}\left\|\chi_{E}\right\|^{p}=\left\|C_{T}\right\|^{p} \mu(E) . \\
& \text { Let } M=\left\|C_{T}\right\|^{p} \text {. Then, } \\
& \qquad \mu T^{-1}(E) \leq M \mu(E) .
\end{aligned}
$$

Conversely, suppose that the condition is true. Then, $\mu T^{-1} \ll$ $\mu$ and hence the Radon-Nikodym derivative $f_{0}$ of $\mu T^{-1}$ with respect to $\mu$ exists and $f_{0} \leq M$ a.e.

Let $f \in \operatorname{Ces}_{p}(X)$. Then,

$$
\begin{aligned}
\left\|C_{T} f\right\|^{p} & =\int_{X}\left(\frac{1}{x} \int_{0}^{x}|(f \circ T)(t)| d \mu(t)\right)^{p} d \mu(x) \\
& \leq \int_{X}\left(\frac{1}{x} \int_{0}^{x}|f| d \mu T^{-1}(t)\right)^{p} d \mu(x) \\
& =\int_{X}\left(\frac{1}{x} \int_{0}^{x}|f| f_{0} d \mu(t)\right)^{p} d \mu(x) \\
& \leq M^{p} \int_{X}\left(\frac{1}{x} \int_{0}^{x}|f(t)| d \mu(t)\right)^{p} d \mu(x) .
\end{aligned}
$$

Therefore, $\left\|C_{T} f\right\| \leq M\|f\|$.

Now, Let $N=\sup _{0<\mu(E)<\infty}\left(\left(\mu\left(T^{-1}(E)\right) / \mu(E)\right)^{p}\right)^{1 / p}$. Then, $\left(\left(\mu\left(T^{-1}(E)\right) / \mu(E)\right)^{p}\right)^{1 / p} \leq N$ for all $E \in s$ and $\mu(E) \neq 0$. Thus $\left(\mu T^{-1}(E)\right)^{p} \leq N^{p}(\mu(E))^{p}$ for all $E \in s$. By the first part of the theorem, we have

$$
\left\|C_{T} f\right\| \leq N\|f\|, \quad \forall f \in \operatorname{Ces}_{p}(X) .
$$


Hence, $\left\|C_{T}\right\|=\sup _{f \neq 0}\left\|C_{T} f\right\| /\|f\| \leq N$. Thus,

$$
\left\|C_{T}\right\| \leq \sup _{0<\mu(E)<\infty}\left(\left(\frac{\mu\left(T^{-1}(E)\right)}{\mu(E)}\right)^{p}\right)^{1 / p} .
$$

On the other hand, Let $M=\left\|C_{T}\right\|=\sup _{f \neq 0}\left\|C_{T} f\right\| /\|f\|$. Thus, $\left\|C_{T} f\right\| /\|f\| \leq M$ for all $f \in \operatorname{Ces}_{p}(X), f \neq 0$. In particular, for $f=\chi_{E}$, such that $0<\mu(E)<\infty, E \in s$, we have $f=$ $\chi_{E} \in \operatorname{Ces}_{p}(X)$ and $\left\|C_{T} \chi_{E}\right\| /\left\|\chi_{E}\right\|=\left(\left(\mu\left(T^{-1}(E)\right) / \mu(E)\right)^{p}\right)^{1 / p} \leq$ $M$.

Therefore,

$$
\sup _{0<\mu(E)<\infty}\left(\left(\frac{\mu\left(T^{-1}(E)\right)}{\mu(E)}\right)^{p}\right)^{1 / p} \leq M=\left\|C_{T}\right\| .
$$

From (10) and (11), we obtain

$$
\left\|C_{T}\right\|=\sup _{0<\mu(E)<\infty}\left(\left(\frac{\mu\left(T^{-1}(E)\right)}{\mu(E)}\right)^{p}\right)^{1 / p} .
$$

Theorem 2. If $C_{T}: \operatorname{Ces}_{p}(X) \rightarrow \operatorname{Ces}_{p}(X)$ is a linear transformation, then $C_{T}$ is continuous.

Proof. Let $\left\{f_{n}\right\}$ and $\left\{C_{T} f_{n}\right\}$ be sequences in $\operatorname{Ces}_{p}(X)$ such that

$$
f_{n} \longrightarrow f, \quad C_{T} f_{n} \longrightarrow g \text { for some } f, g \in \operatorname{Ces}_{p}(X) .
$$

Then, we can find a subsequence $\left\{f_{n_{k}}\right\}$ of $\left\{f_{n}\right\}$ such that

$$
\left\|f_{n_{k}}-f\right\|(t) \longrightarrow 0 \quad \text { for } \mu \text {-almost all } t \in X .
$$

From the nonsingularity of $T$,

$$
\left(\left\|f_{n_{k}}-f\right\| \circ T\right)(t) \longrightarrow 0 \quad \text { for } \mu \text {-almost all } t \in X .
$$

Then, from (13) and (15), we conclude that $C_{T} f=g$. This proves that graph of $C_{T}$ is closed and hence, by closed graph theorem, $C_{T}$ is continuous.

\section{Compactness and Essential Norm of Composition Operators}

This section is devoted to the study of compact composition operators on Cesàro function spaces. A necessary and sufficient condition for a composition operator to be compact is reported in this section. The main aim of this section is to compute the essential norm of the composition operators.

Theorem 3. $L e t C_{T} \in B\left(\operatorname{Ces}_{p}(X)\right)$. Then, $C_{T}$ is compact if and only if $\operatorname{Ces}_{p}\left(X_{\epsilon}, \mu T^{-1}\right)$ is finite dimensional, for each $\epsilon>0$, where

$$
X_{\epsilon}=\left\{x \in X: \frac{d \mu T^{-1}}{d \mu}(x) \geq \epsilon\right\} .
$$

Proof. For $f \in \operatorname{Ces}_{p}(X)$, we have

$$
\begin{aligned}
\left\|C_{T} f\right\|_{\mu} & =\left(\int_{X}\left(\frac{1}{x} \int_{0}^{x}|f \circ T|(t) d \mu(t)\right)^{p} d \mu(x)\right)^{1 / p} \\
& =\left(\int_{X}\left(\frac{1}{x} \int_{0}^{x}|f| d \mu T^{-1}(t)\right)^{p} d \mu(x)\right)^{1 / p} \\
& =\left(\int_{X}\left(\frac{1}{x} \int_{0}^{x}|f| d \mu T^{-1}(t)\right)^{p} d \mu T^{-1}(x)\right)^{1 / p} \\
& =\|f\|_{\mu T^{-1}} \\
& =\|I f\|_{\mu T^{-1}} .
\end{aligned}
$$

Then, $C_{T}$ is compact if and only if $I: \operatorname{Ces}_{p}\left(X_{\epsilon}, \mu T^{-1}\right) \rightarrow$ $\operatorname{Ces}_{p}\left(X_{\epsilon}, \mu T^{-1}\right)$ is a compact operator if and only if $\operatorname{Ces}_{p}\left(X_{\epsilon}, \mu T^{-1}\right)$ is a finite dimensional, where $I$ is the identity operator.

Corollary 4. If $(X, s, \mu)$ is a nonatomic measure space. Then no nonzero composition operator on $\operatorname{Ces}_{p}(X)$ is compact.

Let $X=X_{1} \cup X_{2}$ be the decomposition of $X$ into nonatomic and atomic parts, respectively. If $X_{2}=\phi$ or $\mu(X)=+\infty$ and $X_{2}$ consists of finitely many atoms, then, by Theorem $3, \operatorname{Ces}_{p}(X)$ does not admit a nonzero compact composition operator. Thus, in this case, $\mathscr{K}=\{0\}$ and hence $\left\|C_{T}\right\|_{e}=\left\|C_{T}\right\|$.

Now, we present the main result of this section.

Theorem 5. Let $X_{2}$ consists of finitely many atoms. Suppose that $\operatorname{Ces}_{p}\left(\chi_{\epsilon}, \mu T^{-1}\right)$ is a finite dimensional; that is, $X_{\epsilon}=\{x \in$ $\left.X:\left(d \mu T^{-1} / d \mu\right)(x) \geq \epsilon\right\}$ is a finite dimensional. Let $\alpha=$ $\inf \left\{\epsilon>0: X_{\epsilon}\right.$ is a finite dimensional $\}$. Then,
(i) $\left\|C_{T}\right\|_{e}=0$ iff $\alpha=0$;
(ii) $\left\|C_{T}\right\|_{e} \geq \alpha$ if $0<\alpha \leq 1$;
(iii) $\left\|C_{T}\right\|_{e} \leq \alpha$ if $\alpha>1$.

Proof. (i) Theorem 3 implies that $C_{T}$ is compact if and only if $\alpha=0$. So (i) is the direct consequence of Theorem 3 .

(ii) Suppose that $0<\alpha \leq 1$. Take $0<\epsilon<2 \alpha$ arbitrary. The definition of $\alpha$ implies that $F=\chi_{\alpha-(\epsilon / 2)}$ either contains a nonatomic subset or has infinitely many atoms. If $F$ contains a nonatomic subset, then there are measurable sets $E_{n}, n \in \mathbb{N}$ such that $E_{n+1} \subseteq E_{n} \subseteq F, 0<\mu\left(E_{n}\right)<1 / n$. Define $f_{n}=$ $1 /\left(\mu\left(E_{n}\right)\right)^{1 / p} \chi_{E_{n}}$. Then, $\left\|f_{n}\right\|=1$, for all $n \in \mathbb{N}$. We claim that $f_{n} \rightarrow 0$ weakly. For this, we show that $\int_{X} f_{n} g d \mu \rightarrow 0$, for all $g \in\left(\operatorname{Ces}_{q}(X)\right)$. Let $A \subseteq F$ with $\mu(A)<\infty$ and $g=\chi_{A}$. Then, we have

$$
\begin{aligned}
\left|\int_{X} f_{n} \chi_{A} d \mu\right| & =\frac{1}{\left(\mu\left(E_{n}\right)\right)^{1 / p}} \mu\left(A \cap E_{n}\right) \\
& \leq\left(\frac{1}{n}\right)^{1-(1 / p)} \longrightarrow 0 \quad \text { as } n \longrightarrow \infty .
\end{aligned}
$$

Since simple functions are dense in $\operatorname{Ces}_{q}(X)$, thus $f_{n}$ is proved to converge to zero weakly. Now, assume that $F$ consists of 
infinitely many atoms. Let $\left\{E_{n}\right\}_{n=0}^{\infty}$ be disjoint atoms in $F$. Again, put $f_{n}$ as above. It is easy to see that, for $A \subseteq F$ with $0<\mu(A)<\infty$, we have $\mu\left(A \cap E_{n}\right)=0$ for sufficiently large $n$. So, in both cases, $\int_{X} f_{n} g d \mu \rightarrow 0$. Now, we claim that $\left\|C_{T} f_{n}\right\| \geq \alpha-(\epsilon / 2)$. Since $0<\alpha-(\epsilon / 2)<1$, we see that

$$
\begin{aligned}
\left\|C_{T} f_{n}\right\| & =\left(\int_{X}\left(\frac{1}{x} \int_{0}^{x}\left|C_{T} f_{n}\right| d \mu(t)\right)^{p} d \mu(x)\right)^{1 / p} \\
& =\left(\int_{X}\left(\frac{1}{x} \int_{0}^{x}\left|f_{n}\right| f_{0}(t) d \mu(t)\right)^{p} d \mu(x)\right)^{1 / p} \\
& \geq\left(\int_{X}\left(\frac{1}{x} \int_{0}^{x}\left(\alpha-\frac{\epsilon}{2}\right)\left|f_{n}\right| d \mu(t)\right)^{p} d \mu(x)\right)^{1 / p} \\
& =\left(\alpha-\frac{\epsilon}{2}\right)\left(\int_{X}\left(\frac{1}{x} \int_{0}^{x}\left|f_{n}\right| d \mu(t)\right)^{p} d \mu(x)\right)^{1 / p} \\
& =\alpha-\frac{\epsilon}{2} .
\end{aligned}
$$

Finally, take a compact operator $S$ on $\operatorname{Ces}_{p}(X)$ such that $\| C_{T}-$ $S\|\leq\| C_{T} \|_{e}+(\epsilon / 2)$. Then, we have

$$
\begin{aligned}
\left\|C_{T}\right\|_{e}>\left\|C_{T}-S\right\|-\frac{\epsilon}{2} & \geq\left\|C_{T} f_{n}-S f_{n}\right\|-\frac{\epsilon}{2} \\
& \geq\left\|C_{T} f_{n}\right\|-\left\|S f_{n}\right\|-\frac{\epsilon}{2} \\
& \geq\left(\alpha-\frac{\epsilon}{2}\right)-\left\|S f_{n}\right\|-\frac{\epsilon}{2},
\end{aligned}
$$

for all $n \in \mathbb{N}$. Since a compact operator maps weakly convergent sequences into norm convergent ones, it follows $\left\|S f_{n}\right\| \rightarrow 0$. Hence, $\left\|C_{T}\right\|_{e} \geq \alpha-\epsilon$. Since $\epsilon$ is arbitrary, we obtain $\left\|C_{T}\right\|_{e} \geq \alpha$.

(iii) Let $\alpha>1$ and take $\epsilon>0$ arbitrary. Put $K=X_{\alpha+\epsilon}$. The definition of $\alpha$ implies that $K$ consists of finitely many atoms. So, we can write $K=\left(E_{1}, E_{2}, \ldots, E_{m}\right)$, where $E_{1}, E_{2}, \ldots, E_{m}$ are distinct. Since $\left(L_{\chi_{K}} C_{T} f\right) X=\sum_{i=1}^{m} \chi_{K}\left(E_{i}\right) f\left(T\left(E_{i}\right)\right)$, for all $f \in \operatorname{Ces}_{p}(X)$, hence $L_{\chi_{K}} C_{T}$ has finite rank. Now, let $F \subseteq X \backslash K$ such that $0<\mu(F)<\infty$. Then, we have

$$
\mu T^{-1}(F) \leq(\alpha+\epsilon) \mu(F) .
$$

It follows that $\left\|\chi_{F} \circ T\right\| \leq(\alpha+\epsilon)\left\|\chi_{F}\right\|$. Since simple functions are dense in $\operatorname{Ces}_{p}(X)$. We obtain

$$
\sup _{\|f\| \leq 1}\left\|\chi_{X \backslash K} f \circ T\right\| \leq \sup _{\|f\| \leq 1}\left\|\chi_{X \backslash K} f\right\| \leq \alpha+\epsilon .
$$

Finally, Since $L_{\chi_{K}} C_{T}$ is a compact operator, we get

$$
\begin{aligned}
\left\|C_{T}-L_{\chi_{K}} C_{T}\right\| & =\sup _{\|f\| \leq 1}\left\|\left(1-\chi_{K}\right) C_{T} f\right\| \\
& =\sup _{\|f\| \leq 1}\left\|\chi_{X \backslash K} C_{T} f\right\| \\
& \leq \alpha+\epsilon .
\end{aligned}
$$

It follows that $\left\|C_{T}\right\|_{e} \leq \alpha+\epsilon$ and, consequently, $\left\|C_{T}\right\|_{e} \leq \alpha$.
Example 6. Let $X=(-\infty, 0] \cup \mathbb{N}$, where $\mathbb{N}$ is the set of natural numbers. Let $\mu$ be the Lebesgue measure on $(-\infty, 0]$ and $\mu(\{n\})=1 / 2^{n}$ if $n \in \mathbb{N}$. Define $T: \mathbb{N} \rightarrow \mathbb{N}$ as $T(1)=T(2)=T(3)=1, T(4)=2, T(5)=T(6)=3$, and $T(2 n+1)=5$, for $n \geq 3$.

Consider $T(2 n)=2 n-2$, for $n \geq 4$, and $T(x)=5 x$, for all $x \in(-\infty, 0]$. Then, we can easily calculate $\left\|C_{T}\right\|_{e}=$ $3^{-1 / p}$ on $\operatorname{Ces}_{p}(X)$ for $1<p<\infty$.

\section{Fredholm and Isometric Composition Operators}

In this section, we first establish a condition for the composition operators to have closed range and then we make the use of it to characterize the Fredholm composition operators. We also make an attempt to compute the adjoint of the composition operators.

Holder's inequality for Cesàro measurable function spaces is that, if $f \in \operatorname{Ces}_{p}(X)$ and $g \in \operatorname{Ces}_{q}(X)$ such that $1 / p+1 / q=1$, then

$$
\int f g d \mu \leq\|f\|_{p}\|g\|_{q}
$$

We find that every $g \in \operatorname{Ces}_{q}(X)$ gives rise to a bounded linear functional $F g \in\left(\operatorname{Ces}_{p}(X)\right)^{*}$ which is defined as

$$
F g(f)=\int f g d \mu, \quad \text { for every } f \in \operatorname{Ces}_{p}(X) \text {. }
$$

For each $f \in \operatorname{Ces}_{p}(X)$, there exists a unique $T^{-1}(s)$ measurable function $E(f)$ such that $\int g f d \mu=\int g E(f) d \mu$ for $T^{-1}(s)$ measurable function $g$ for which the left integral exists. The function $E(f)$ is called conditional expectation of $f$ with respect to the $\sigma$-algebra $T^{-1}(s)$. The operator $P_{T}: \operatorname{Ces}_{p}(X) \rightarrow$ $\operatorname{Ces}_{p}(X)$ defined by $P_{T} f=f_{0} E(f) \circ T^{-1}$ is called the Frobenius Perron operator where $E(f) \circ T^{-1}=g$ if and only if $E(f)=g \circ T$.

Theorem 7. Let $C_{T} \in B\left(\operatorname{Ces}_{p}(X)\right)$. Then $C_{T}$ has closed range if and only if there exists $\delta>0$ such that $f_{0}(x) \geq \delta$ for $\mu$-almost all $x \in \operatorname{supp} f_{0}=S$.

Proof. If $f_{0}(x) \geq \delta$ for $\mu$-almost all $x \in S$, then, for $\eta=$ $\min (\delta, 1 / \delta) \leq 1$,

$$
\begin{aligned}
1 & \geq\left(\int_{X}\left(\frac{1}{x} \int_{0}^{x} \frac{\left|C_{T} f\right|}{\left\|C_{T} f\right\|} d \mu(t)\right)^{p} d \mu(x)\right)^{1 / p} \\
& =\left(\int_{X}\left(\frac{1}{x} \int_{0}^{x} f_{0} \frac{|f|}{\left\|C_{T} f\right\|} d \mu(t)\right)^{p} d \mu(x)\right)^{1 / p} \\
& \geq\left(\int_{X}\left(\frac{1}{x} \int_{0}^{x} \frac{\eta|f|}{\left\|C_{T} f\right\|} d \mu(t)\right)^{p} d \mu(x)\right)^{1 / p} .
\end{aligned}
$$

Hence, $\left\|C_{T} f\right\| \geq \eta\|f\|$, for all $f \in \operatorname{Ces}_{p}(S)$, so that $C_{T}$ has closed range. 
Conversely, suppose that $C_{T}$ has closed range. Then there exists $\delta \geq 0$ such that

$$
\left\|C_{T} f\right\| \geq \delta\|f\|
$$

for every $f \in \operatorname{Ces}_{p}(S)$. Choose a positive integer $n$ such that $1 / n<\delta$. If the set $E=\left\{x \in X: f_{0}(x)<1 / n\right\}$ has positive measure, then, for a given measurable subset $F \subset \operatorname{supp} f_{0}$ such that $0<\mu(F)<\infty$, we have

$$
\mu T^{-1}(E)<\frac{1}{n} \mu(E)
$$

or equivalently

$$
\left\|C_{T} \chi_{E}\right\| \leq \frac{1}{n}\left\|\chi_{E}\right\|
$$

This contradicts inequality (27). Hence, $f_{0}$ is bounded away from zero on $\operatorname{supp} f_{0}$.

Theorem 8. Let $C_{T} \in B\left(\operatorname{Ces}_{p}(X)\right)$. Then, $\operatorname{ker} C_{T}^{*}$ is either zero-dimensional or infinite dimensional.

Proof. Suppose $0 \neq g \in \operatorname{ker} C_{T}^{*}$. Then $E=\operatorname{supp} g$ is a set of nonzero measure. Now we can partition $E$ into a sequence $\left\{E_{n}\right\}$ of measurable sets, $0<\mu\left(E_{n}\right)<\infty$. We show that $g \chi_{E_{n^{\circ}} \circ} \in \operatorname{ker} C_{T}^{*}$. Consider

$$
\begin{aligned}
C_{T}^{*}\left(g \chi_{E_{n} \circ T}\right)(f) & =\int_{E}\left(g \chi_{E_{n} \circ T}\right)\left(C_{T} f\right) d \mu \\
& =\int_{E} g C_{T}\left(\chi_{E_{n}} f\right) d \mu=0 .
\end{aligned}
$$

Hence, if ker $C_{T}^{*}$ is not zero-dimensional, it is infinite dimensional.

Corollary 9. Let $C_{T} \in B\left(\operatorname{Ces}_{p}(X)\right)$. Then, $C_{T}$ is injective if and only if $T$ is surjective.

Theorem 10. Let $C_{T} \in B\left(\operatorname{Ces}_{p}(X)\right)$. Then, $C_{T}$ has dense range if and only if $T^{-1}(s)=s$.

Proof. Suppose that $C_{T}$ has dense range. Let $E \in s$ such that $\chi_{E} \in \operatorname{Ces}_{p}(X)$. Then, there exists $\left\{f_{n}\right\} \subset \operatorname{Ces}_{p}(X)$ such that $C_{T} f_{n} \rightarrow \chi_{E}$. Now, we can find a subsequence $\left\{f_{n_{k}}\right\}$ of $\left\{f_{n}\right\}$ such that $C_{T} f_{n_{k}} \rightarrow \chi_{E}$ a.e. Now, each $C_{T} f_{n_{k}}$ is measurable with respect to $T^{-1}(s)$. Therefore, $\chi_{E}$ is measurable with respect to $T^{-1}(s)$ so that $\chi_{E}=\chi_{T^{-1}(F)}$. Hence, $T^{-1}(s)=s$ a.e.

Conversely, suppose $T^{-1}(s)=s$ a.e. If $E \in s, 0<\mu(E)<$ $\infty$, then there exists $F \in s$ such that $\mu\left(T^{-1}(F) \Delta E\right)=0$. Since $X$ is $\sigma$-finite, we can find an increasing sequence $\left\{F_{n}\right\}$ of sets of finite measure $F_{n} \uparrow F$ or $T^{-1}(F) \backslash T^{-1}\left(F_{n}\right) \downarrow 0$. Hence, for given $\epsilon>0$, there exists a positive integer $n_{0}$ such that $\mu\left(T^{-1}\left(F \backslash F_{n}\right)\right)<\epsilon$ for every $n \geq n_{0}$. Hence,

$$
\begin{aligned}
\left\|C_{T} \chi_{F}-C_{T} \chi_{F_{n}}\right\| & =\left\|C_{T}\left(\chi_{F \backslash F_{n}}\right)\right\| \\
& \left.=\| \chi_{T^{-1}\left(F \backslash F_{n}\right.}\right) \| \\
& =\left(\int_{X}\left(\frac{1}{x} \int_{0}^{x} \chi_{T^{-1}\left(F \backslash F_{n}\right)} d \mu(t)\right)^{p} d \mu(x)\right)^{p} \\
& <\epsilon,
\end{aligned}
$$

for all $n \geq n_{0}$. Then $\chi_{F}=\chi_{T^{-1}(F)} \in \overline{\operatorname{ran} C_{T}}$. This proves that $C_{T}$ has dense range.

Theorem 11. Let $C_{T} \in B\left(\operatorname{Ces}_{p}(X)\right)$. Then, $C_{T}$ is Fredholm if and only if $C_{T}$ is invertible.

Proof. Assume that $C_{T}$ is Fredholm. In view of Theorem 8, $\operatorname{ker} C_{T}$ and $\operatorname{ker} C_{T}^{*}$ are zero-dimensional so that $C_{T}$ is injective and $T^{-1}(s)=s$ a.e. Therefore, by Theorem $10, C_{T}$ has dense range. Since ran $C_{T}$ is closed, so $C_{T}$ is surjective. This proves the invertibility of $C_{T}$. The proof of the converse part is obvious.

Corollary 12. Let $C_{T} \in B\left(\operatorname{Ces}_{p}(X)\right)$. Then, $C_{T}$ is an isometry if and only if $T$ is measure preserving.

Proof. If $T$ is measure preserving, then $f_{0}=1$ a.e. Therefore,

$$
\begin{aligned}
\left\|C_{T} f\right\| & =\left(\int_{X}\left(\frac{1}{x} \int_{0}^{x}|f \circ T|(t) d \mu(t)\right)^{p} d \mu(x)\right)^{1 / p} \\
& =\left(\int_{X}\left(\frac{1}{x} \int_{0}^{x} f_{0}|f| d \mu(t)\right)^{p} d \mu(x)\right)^{1 / p} \\
& =\left(\int_{X}\left(\frac{1}{x} \int_{0}^{x}|f| d \mu(t)\right)^{p} d \mu(x)\right)^{1 / p} \\
& =\|f\| .
\end{aligned}
$$

Hence, $C_{T}$ is an isometry. Conversely, if $C_{T}$ is an isometry, then

$$
\left\|C_{T} \chi_{E}\right\|=\left\|\chi_{E}\right\|
$$

This implies that $\mu\left(T^{-1}(E)\right)=\mu(E)$ for $E \in s$. Hence, $f_{0}=$ 1 a.e.

Theorem 13. Let $C_{T} \in B\left(\operatorname{Ces}_{p}(X)\right)$. Then, $C_{T}^{*}=P_{T}$. 
Proof. Let $A \in s$ such that $0<\mu(A)<\infty$. For $g \in \operatorname{Ces}_{q}(X)$,

$$
\begin{aligned}
\left(C_{T}^{*} F g\right)\left(\chi_{A}\right) & =F g\left(C_{T} \chi_{A}\right) \\
& =\int C_{T} \chi_{A} g d \mu \\
& =\int \chi_{A} \circ T g d \mu \\
& =\int \chi_{A} E(g) \circ T^{-1} f_{0} d \mu \\
& =F_{E(g) \circ T^{-1} f_{0}}\left(\chi_{A}\right) .
\end{aligned}
$$

Hence, $C_{T}^{*} F g=F_{E(g) \circ T^{-1}} f_{0}$. After identifying $g \in \operatorname{Ces}_{q}(X)$ with $F g \in\left(\operatorname{Ces}_{p}(X)\right)^{*}$, we can write $C_{T}^{*} g=E(g) \circ T^{-1} f_{0}=$ $P_{T} \mathfrak{g}$.

\section{Conflict of Interests}

The authors declare that there is no conflict of interests regarding the publication of this paper.

\section{References}

[1] S. V. Astashkin and L. Maligranda, "Structure of Cesàro function spaces," Indagationes Mathematicae, vol. 20, no. 3, pp. 329379, 2009.

[2] J.-S. Shiue, "A note on Cesàro function space," Tamkang Journal of Mathematics, vol. 1, no. 2, pp. 91-95, 1970.

[3] B. D. Hassard and D. A. Hussein, "On Cesàro function spaces," Tamkang Journal of Mathematics, vol. 4, pp. 19-25, 1973.

[4] P. W. Sy, W. Y. Zhang, and P. Y. Lee, "The dual of Cesàro function spaces," Glasnik Matematički, vol. 22, no. 1, pp. 103-112, 1987.

[5] B. I. Korenblyum, S. G. Kreĭn, and B. Y. Levin, "On certain nonlinear questions of the theory of singular integrals," Proceedings of the National Academy of Sciences of the United States of America, vol. 62, pp. 17-20, 1948.

[6] E. Sawyer, "Boundedness of classical operators on classical Lorentz spaces," Studia Mathematica, vol. 96, no. 2, pp. 145-158, 1990.

[7] S. V. Astashkin and L. Maligranda, "Cesàro function spaces fail the fixed point property," Proceedings of the American Mathematical Society, vol. 136, no. 12, pp. 4289-4294, 2008.

[8] S. V. Astashkin and L. Maligranda, "Rademacher functions in Cesàro type spaces," Studia Mathematica, vol. 198, no. 3, pp. 235-247, 2010.

[9] A. C. Zaanen, Integration, North-Holland, 2nd edition, 1967.

[10] E. Schrljeder, "Uber iteratierte funcktionen," Journal of Mathematical Analysis, vol. 3, pp. 296-322, 1871.

[11] G. Köenigs, "Recherches sur le Integrable de certcuns equarions frontionalles," Annales Scientifiques de l'École Normale Supérieure, vol. 1, pp. 3-41, 1884.

[12] J. E. Littlewood, "On inequalities in the theory of functions," Proceedings of the London Mathematical Society, vol. 23, no. 1, pp. 481-519, 1925.

[13] B. O. Koopman, "Hamiltonian systems and transformations in Hilbert spaces," Proceedings of the National Academy of Sciences of the United States of America, vol. 17, pp. 315-318, 1931.
[14] E. A. Nordgren, "Composition operators," Canadian Journal of Mathematics, vol. 20, pp. 442-449, 1968.

[15] Y. Cui, H. Hudzik, R. Kumar, and L. Maligranda, "Composition operators in Orlicz spaces," Journal of the Australian Mathematical Society, vol. 76, no. 2, pp. 189-206, 2004.

[16] R. G. Douglas, Banach Algebra Techniques in Operator Theory, Academic Press, New York, NY, USA, 1972.

[17] W. E. Hornor and J. E. Jamison, "Isometrically equivalent composition operators," Contemporary Mathematics, vol. 213, pp. 65-72, 1996.

[18] M. R. Jabbarzadeh, "A note on weighted composition operators on measurable function spaces," Journal of the Korean Mathematical Society, vol. 41, no. 1, pp. 95-105, 2004.

[19] B. S. Komal and R. S. Pathania, "Composition operators on a space of operators," Indian Journal of Mathematics, vol. 33, no. 1, pp. 11-17, 1991.

[20] A. Kumar, "Fredholm composition operators," Proceedings of the American Mathematical Society, vol. 79, no. 2, pp. 233-236, 1980.

[21] B. D. MacCluer, "Fredholm composition operators," Proceedings of the American Mathematical Society, vol. 125, no. 1, pp. 163166, 1997.

[22] E. A. Nordgren, Composition Operators on Hilbert Spaces, vol. 693 of Lectures Notes in Math, Springer, New York, NY, USA, 1978.

[23] K. Raj, A. K. Sharma, and A. Kumar, "Weighted composition operators on Musielak-Orlicz spaces," Ars Combinatoria, vol. 107, pp. 431-439, 2012.

[24] K. Raj and S. K. Sharma, "Composition operators on MusielakOrlicz spaces of Bochner type," Mathematica Bohemica, vol. 137, no. 4, pp. 449-457, 2012.

[25] W. C. Ridge, Composition operators [Ph.D. thesis], Indiana University, 1969.

[26] R. K. Singh and J. S. Manhas, Composition Operators on Function Spaces, North-Holland, 1993.

[27] H. Takagi and K. Yokouchi, "Multiplication and composition operators between two $L^{p}$-spaces," Contemporary Mathematics, vol. 232, pp. 321-338, 1999.

[28] A. Lambert, "Localising sets for sigma-algebras and related point transformations," Proceedings of the Royal Society of Edinburgh A, vol. 118, no. 1-2, pp. 111-118, 1991.

[29] J. H. Shapiro, “The essential norm of a composition operator," Annals of Mathematics, vol. 125, no. 2, pp. 375-404, 1987.

[30] M. R. Jabbarzadeh, "The essential norm of a composition operator on Orlicz spaces," Turkish Journal of Mathematics, vol. 34, no. 4, pp. 537-542, 2010.

[31] A. Montes-Rodríguez, "The essential norm of a composition operator on Bloch spaces," Pacific Journal of Mathematics, vol. 188, no. 2, pp. 339-351, 1999.

[32] H. Takagi, T. Miura, and S.-E. Takahasi, "Essential norms and stability constants of weighted composition operators on $C(X)$," Bulletin of the Korean Mathematical Society, vol. 40, no. 4, pp. 583-591, 2003.

[33] L. Zheng, "The essential norms and spectra of composition operators on $H^{\infty}$," Pacific Journal of Mathematics, vol. 203, no. 2, pp. 503-510, 2002. 


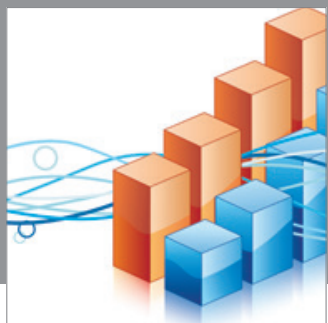

Advances in

Operations Research

mansans

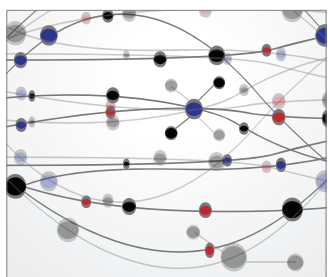

The Scientific World Journal
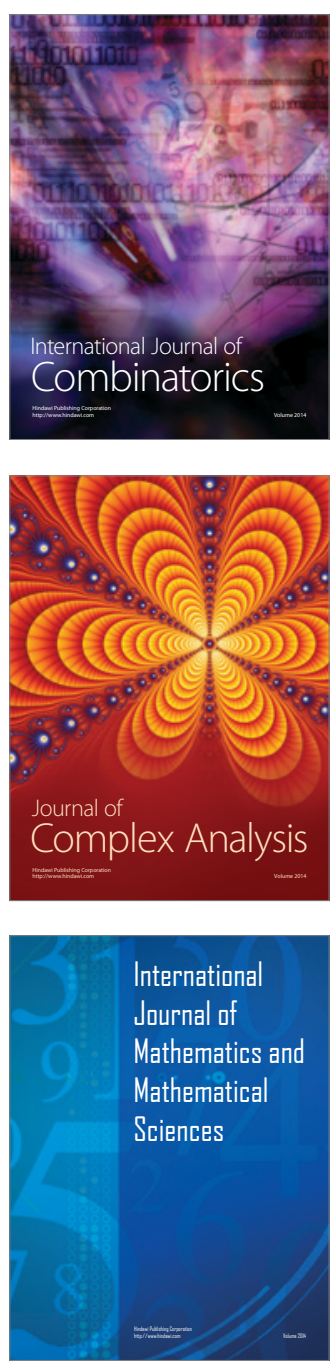
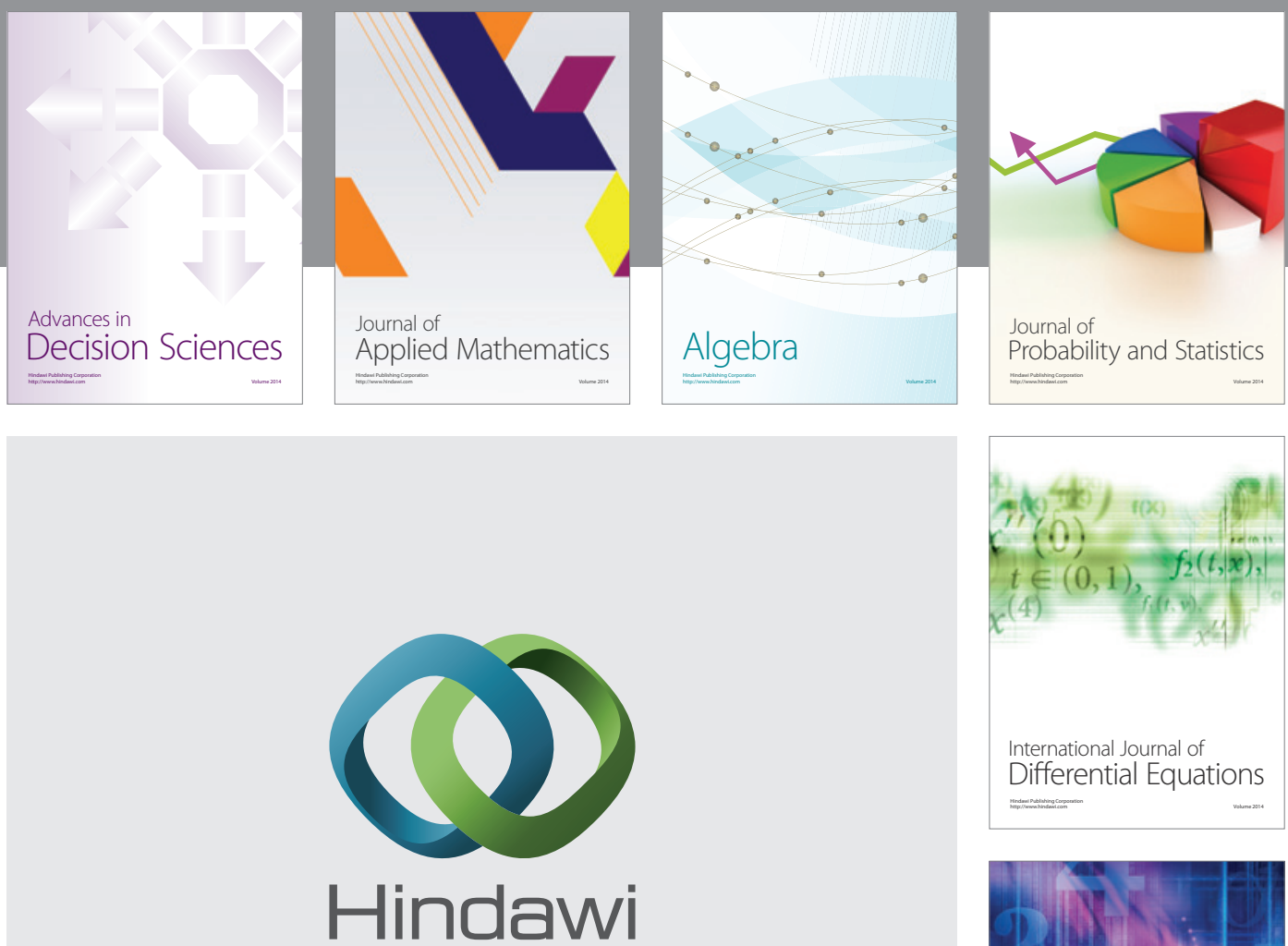

Submit your manuscripts at http://www.hindawi.com
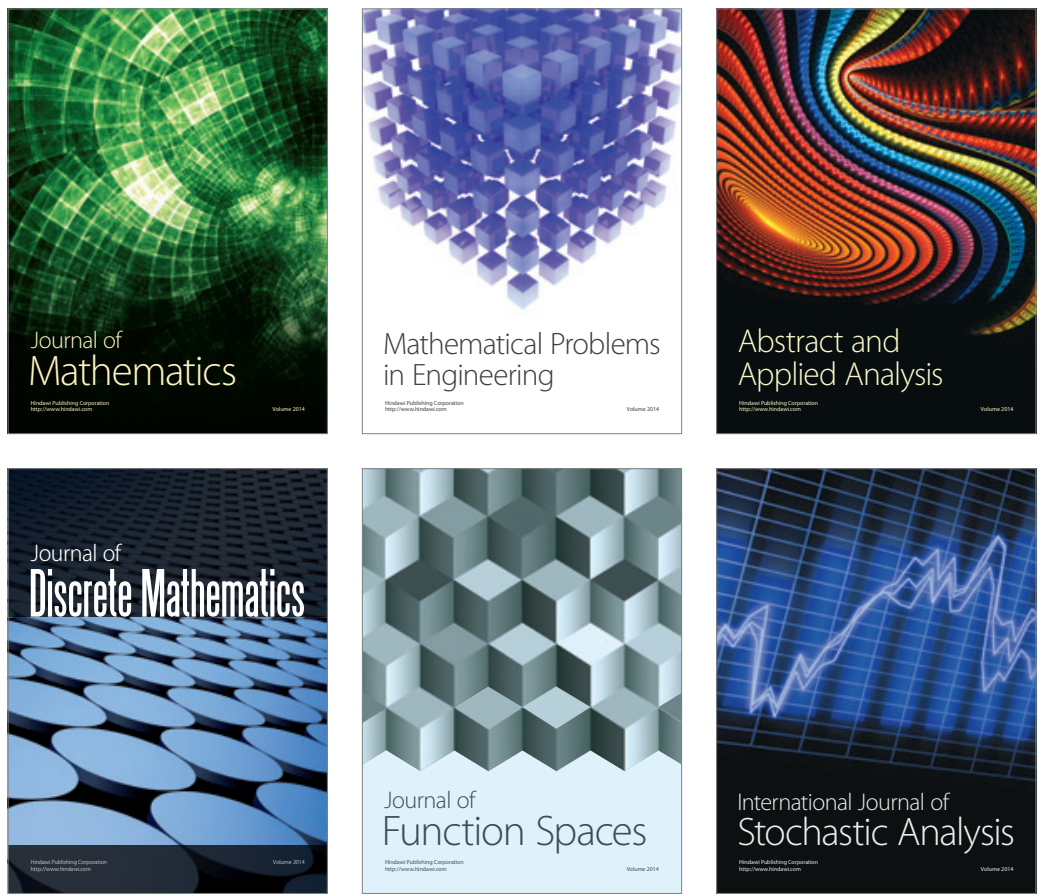

Journal of

Function Spaces

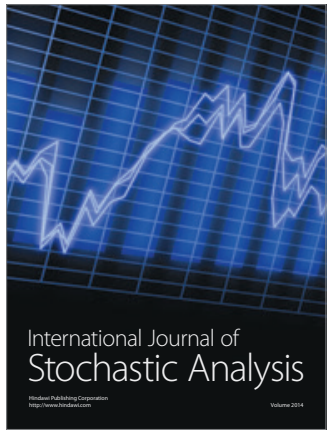

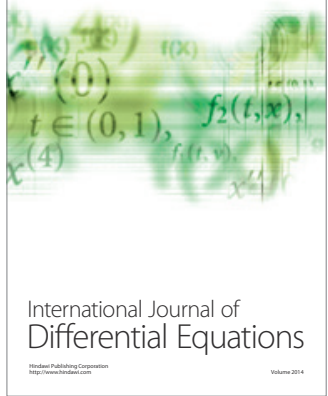
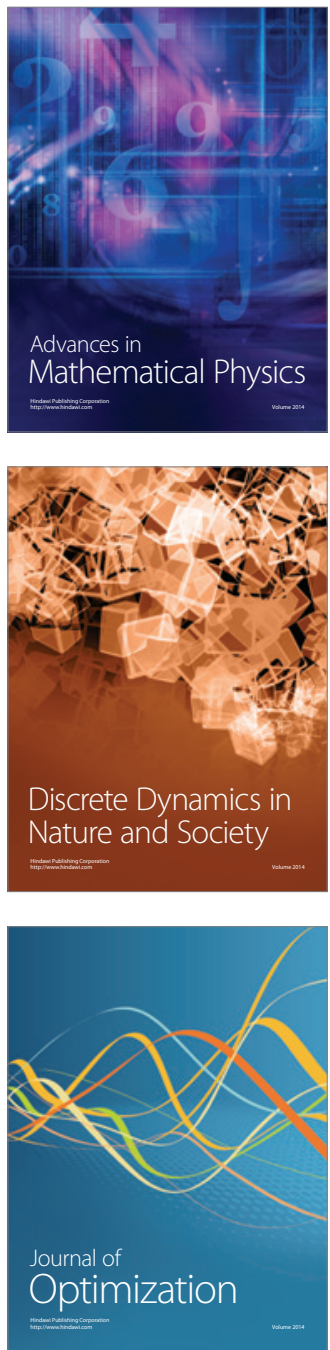